\title{
BMJ Open Perceived burden in dealing with different rare diseases: a qualitative focus group study
}

\author{
Natalie Uhlenbusch (D) , Bernd Löwe, Miriam K Depping
}

To cite: Uhlenbusch N, Löwe B, Depping MK. Perceived burden in dealing with different rare diseases: a qualitative focus group study. BMJ Open 2019;9:e033353. doi:10.1136/ bmjopen-2019-033353

\section{- Prepublication history and} additional material for this paper are available online. To view these files, please visit the journal online (http://dx.doi org/10.1136/bmjopen-2019033353).

Received 31 July 2019 Revised 28 0ctober 2019 Accepted 05 December 2019

Check for updates

(c) Author(s) (or their employer(s)) 2019. Re-use permitted under CC BY-NC. No commercial re-use. See rights and permissions. Published by BMJ.

Department of Psychosomatic Medicine and Psychotherapy, University Medical Center Hamburg-Eppendorf, Hamburg, Germany

Correspondence to Natalie Uhlenbusch; n.uhlenbusch@uke.de

\section{ABSTRACT}

Objectives There are more than 6000 heterogeneous rare diseases and little is known about shared experiences of affected individuals in everyday life and healthcare. Objective of this study was to explore perceived burden of patients with rare chronic diseases and identify commonalities and differences in the experiences of patients with four heterogeneous conditions.

Design A qualitative focus group study.

Setting In four separate and diagnostically homogeneous focus groups, we asked patients about the perceived burden of living with their rare disease. The focus groups took place at a university medical centre in Germany. Participants Individuals with neurofibromatosis type 1 $(n=4)$, primary sclerosing cholangitis $(n=5)$, pulmonary arterial hypertension $(n=4)$ and Marfan syndrome $(n=5)$. Results We identified five main themes: medical problems, psychological burden, problems with the healthcare system, constraints and interpersonal problems. While medical problems differed widely between the diagnostic groups, patients with different conditions independently reported many common problems including psychological burden, constraints in professional, personal and daily life, stigmatisation and others lacking understanding. Shared problems pertaining to the healthcare system seem related to the rarity of the conditions (eg, limited access to adequate care, lack of knowledge).

Conclusions Despite clinical heterogeneity of rare diseases, affected individuals have many common experiences. Some of these experiences may resemble the burden of living with a chronic disease. However, patients reported aspects, which seem to be specific for rare chronic diseases. Generic interventions targeting shared burdens among patients with different diseases could provide adequate treatment in light of finite healthcare resources.

\section{INTRODUCTION}

Rare diseases by definition affect less than one in 2000 individuals. It is estimated that there exist more than 6000 heterogeneous rare diseases. Although each rare disease affects only a small number of patients, more than 30 million individuals in Europe are affected by any rare disease. The majority of rare diseases are chronic and are associated with high somatic symptom severity, reduced

\section{Strengths and limitations of this study}

To our knowledge, this is the first study systematically examining and comparing the perceived burden of patients with different rare diseases in order to identify shared aspects.

- The qualitative study design allows gaining an indepth insight into the experiences of patients with rare diseases with the goal of understanding the burdens that are specific for the relatively understudied patient population.

- While the small sample size and focus on only four specific rare diseases limits the generalisability of the results, the clearly defined study aim within a specific patient population ensures a high information power.

- The results can help to develop generic interventions to provide psychosocial support for patients with different rare diseases concurrently.

life expectancy and decreased quality of life. Additionally, the low prevalence of rare diseases results in a variety of difficulties that individuals are confronted with. These include lack of knowledge about the diseases, delay in diagnosis and limited access to adequate treatment. Furthermore, the rarity of each condition can lead to a lack of contact to peers with the same disease and feelings of isolation. ${ }^{1}$

These burdens can be a risk to mental health. In a recent quantitative study, high percentages of patients with different rare diseases showed increased levels of depression and anxiety symptoms. ${ }^{2}$ Depression and anxiety in addition to a chronic disease are associated with reduced quality of life and can negatively affect the course of the chronic illness. ${ }^{3}{ }^{4}$ To improve overall health and provide comprehensive treatment, it is crucial to understand the experiences of patients with rare diseases and identify risk and protective factors. ${ }^{5}$

Despite the heterogeneity of the conditions, patients with rare diseases might have many common experiences. In a recent 
systematic review of qualitative studies with each focusing on a specific single rare disease or group of rare diseases, patients described similar problems. These include constraints (e.g., at work), psychological burden, stigmatisation, lack of knowledge and lack of available treatment options. ${ }^{5}$ Common experiences among patients with different diseases could be addressed in health promotion services and concurrently provide patients with different rare diseases access to comprehensive treatment. This could overcome the obstacle that it is practically impossible to develop disease-specific psychosocial interventions for every single rare disease.

However, there is a lack of research on commonalities and differences in the experiences of patients with different rare diseases. ${ }^{5}$ To our knowledge, no studies have investigated the common experiences of people with heterogeneous rare diseases by directly comparing them. Further, it is not understood whether there are potential common experiences specific for rare chronic conditions in contrast to universal experiences of patients with chronic diseases. Information about common and differing experiences can help identify risk and protective factors and inform clinical practice. ${ }^{5}$ Objective of this study was to explore the perceived burden of living with a rare chronic disease and identify commonalities and differences in the experiences of patients with four heterogeneous conditions in a qualitative study design.

\section{METHODS}

\section{Participants}

This study was a qualitative focus group study. We included patients with one of the following four rare diseases: neurofibromatosis type 1, primary sclerosing cholangitis, pulmonary arterial hypertension and Marfan syndrome. Neurofibromatosis type 1 (prevalence:1-5/10.000) is a genetic disease whose symptoms include the growth of partly visible tumours in the skin, changes in pigmentation and tumours in the brain, along nerves and other body parts. ${ }^{6}$ Primary sclerosing cholangitis (prevalence:1-9/100.000) manifests itself by inflammation, scarring and narrowing of the bile ducts and a resultant build-up of bile in the liver. Symptoms include tiredness, abdominal pain and itchiness. In later stages, the condition can cause jaundice, an enlarged spleen and liver cirrhosis/failure. Furthermore, the majority of the patients suffer from comorbidity with ulcerative colitis. ${ }^{7}$ Pulmonary arterial hypertension (prevalence:1-9/100.000) is characterised by an increased blood pressure in the pulmonary artery with symptoms such as shortness of breath and fainting spells. ${ }^{8}$ Marfan syndrome (prevalence:1-5/10.000) is a disease of the connective tissue affecting heart and blood vessels, bones, eyes, skin, nervous system and lungs. ${ }^{9}$ All four conditions are severe chronic conditions assumed to affect quality of life and are treated in specialised outpatient clinics at the University Medical Center Hamburg-Eppendorf. We recruited patients via these outpatient clinics and via patient organisations and self-help groups. Inclusion criteria were one of the abovementioned diagnoses, written informed consent, a minimum age of 16 years, sufficient German knowledge and the possibility to reach the study location. Each participant received an incentive of 20 Euros (approximately US $\$ 23$ at that time).

\section{Procedure}

We conducted four focus groups with diagnostically homogeneous groups of patients affected by either neurofibromatosis type 1 , primary sclerosing cholangitis, pulmonary arterial hypertension or Marfan syndrome. A semistructured interview guide was developed by the research group and discussed with researchers and clinicians in our department beforehand. The interview guide contained questions about problems in living with a rare disease as well as coping mechanisms. This article focuses on problems, which were reported responding to the following questions: 1. Which aspects of the disease do you perceive as particularly burdensome? 2. In what way does the disease impede your daily life? 3. Which consequences does the disease have on your social life, your work or other areas of your life? All focus groups were conducted using the same interview guide.

Eligible patients received printed information flyers about the study. Contact between the research team and the participants before the focus groups was limited to arrangements regarding date and place. No information about the researchers such as research interests or professional background was given. Participants were informed about the study's purpose.

The focus groups took place in a meeting room at the University Medical Center Hamburg-Eppendorf. The average duration of the focus groups was $\mathrm{M}=87.25 \mathrm{~min}$ $(\mathrm{SD}=15.84$, range $68-103 \mathrm{~min})$. One researcher (MKD, woman, psychologist, senior researcher) led the discussions asking questions with another researcher assisting (NU, woman, psychologist, junior researcher). All participants received and signed a written informed consent form. We collected data using audio recording. A research assistant (AW, woman, graduate student in psychology) took field notes during all sessions. The same research assistant transcribed the audio records verbatim. Transcripts were not returned to participants for comments or corrections. All team members were occupied at the Department of Psychosomatic Medicine at the University Medical Center Hamburg-Eppendorf. We used the Consolidated criteria for Reporting Qualitative research checklist to ensure that our study meets the criteria for qualitative research.

\section{Data analysis}

We evaluated the data using qualitative content analysis. ${ }^{10}$ Two team members (NU and AW) inductively and independently built categories according to the study aim (to identify perceived burden of living with a rare disease). The two team members read all four pseudonymised transcripts to identify reoccurring themes. These themes 
were then used to build the category system. The category system was discussed and revised several times with a third researcher (MKD). We identified superordinate themes and built main categories. Two independent team members (LH, woman, medical student; JD, woman, undergraduate student in psychology) who participated neither in the focus groups nor in the development of the coding scheme deductively coded the four transcripts using the final category system. All inconsistencies and the final coding were discussed with a third researcher (NU) until reaching agreement. To identify differences and commonalities between different diagnoses, we analysed and compared which topics were brought up in each diagnostic group. We used the software MAXQDA V.12.2.0 to support the coding process.

\section{Patient and public involvement}

This study is part of the research project 'patients for patients' aiming to develop and evaluate a disease management programme for patients with rare chronic diseases. We closely cooperate with a non-profit alliance (ACHSE e.V.) functioning as umbrella organisation for patient associations for rare diseases. ACHSE e.V. consulted the development, planning and implementation of the project by representing patients' interests. Further, we closely cooperate with patient associations directly who supported our recruitment process and help disseminate our results on their websites and in the associations' patient magazines.

\section{RESULTS}

\section{Sample}

Eighteen patients with either neurofibromatosis type 1 $(n=4)$, primary sclerosing cholangitis $(n=5)$, pulmonary arterial hypertension $(n=4)$ or Marfan syndrome $(n=5)$ took part in four diagnostically homogeneous focus groups. The mean age was $\mathrm{M}=49.82$ years $(\mathrm{SD}=14.75$, range 25-77) and $n=11$ participants were women. One participant did not report his age.

\section{Topics brought up by the participants}

Following the inductive procedure described above, we identified five main themes of burden: medical problems, psychological burden, problems concerning the healthcare system, constraints and interpersonal problems.

\section{Medical problems}

Patients reported a variety of burdening medical problems and somatic symptoms. The majority of these symptoms were specific for certain diagnoses, which reflects the clinical heterogeneity of the four diseases. For instance, only patients with neurofibromatosis type 1 mentioned epilepsy and the growth of tumours, only patients with primary sclerosing cholangitis named itching and cholestasis, and only patients with pulmonary arterial hypertension reported to be affected by problems of the respiratory system and thrombosis.
Table 1 Medical problems and number of mentions (total and by diagnostic group)

\begin{tabular}{|c|c|c|c|c|c|}
\hline Medical problems & NF1 & PSC & PAH & MFS & Tota \\
\hline \multicolumn{6}{|c|}{ Aspects related to specific diagnoses } \\
\hline Tumours & 7 & & & & 7 \\
\hline $\begin{array}{l}\text { Problems of the } \\
\text { respiratory system }\end{array}$ & & & 6 & & 6 \\
\hline Thrombosis & & & 4 & & 4 \\
\hline Epilepsy & 3 & & & & 3 \\
\hline Aneurysms & & & & 2 & 2 \\
\hline Coeliac disease & & 2 & & & 2 \\
\hline Itching & & 2 & & & 2 \\
\hline Cholestasis & & 2 & & & 2 \\
\hline Incontinence & 2 & & & & 2 \\
\hline Low body weight & & & & 1 & 1 \\
\hline $\begin{array}{l}\text { Restriction of renal } \\
\text { function }\end{array}$ & & & & 1 & 1 \\
\hline Endocarditis & & & & 1 & 1 \\
\hline Iron deficiency & & 1 & & & 1 \\
\hline $\begin{array}{l}\text { Neurological } \\
\text { disturbances of the } \\
\text { sensitivity }\end{array}$ & 1 & & & & 1 \\
\hline $\begin{array}{l}\text { Brief reactive } \\
\text { psychosis }\end{array}$ & & & & 1 & 1 \\
\hline
\end{tabular}

Shared aspects named by two diagnostic groups

\begin{tabular}{|c|c|c|c|c|}
\hline Fatigue & & 3 & 5 & \\
\hline $\begin{array}{l}\text { Hormonal } \\
\text { abnormalities }\end{array}$ & & 5 & & 3 \\
\hline Disfigurement & 4 & & & 2 \\
\hline $\begin{array}{l}\text { Neurological } \\
\text { disturbances of the } \\
\text { motor function }\end{array}$ & 4 & & 1 & \\
\hline Damages from surgery & 1 & & & 3 \\
\hline Skin problems & 1 & & & 2 \\
\hline
\end{tabular}

Shared aspects named by three diagnostic groups

\begin{tabular}{|c|c|c|c|c|}
\hline Diarrhoea & 1 & 9 & 1 & \\
\hline Limited eyesight & 2 & & 1 & 5 \\
\hline Pain & 1 & 1 & & 3 \\
\hline $\begin{array}{l}\text { Side effects of } \\
\text { medication }\end{array}$ & & 1 & 1 & 1 \\
\hline
\end{tabular}

Numbers represent the total number of mentions and are displayed for each diagnosis individually as well as across all diagnostic groups; grey cells represent categories, which were mentioned at least once in a diagnostic group.

MFS, Marfan syndrome; NF1, neurofibromatosis type 1; $\mathrm{PAH}$, pulmonary arterial hypertension; PSC, primary sclerosing cholangitis.

However, some medical problems such as pain, diarrhoea or side effects of medication were shared among different diagnoses. No aspect was brought up by all four diagnostic groups. Table 1 shows all named medical problems and the number of mentions for 
Table 2 Psychological burdens and number of mentions (total and by diagnostic group)

\begin{tabular}{|c|c|c|c|c|c|}
\hline Psychological burden & NF1 & PSC & PAH & MFS & Total $N$ \\
\hline \multicolumn{6}{|c|}{ Aspects related to specific diagnoses } \\
\hline Rumination & & 3 & & & 3 \\
\hline Avolition & & 2 & & & 2 \\
\hline Suicidal thoughts & & & & 2 & 2 \\
\hline $\begin{array}{l}\text { Using disease as } \\
\text { excuse for other } \\
\text { problems }\end{array}$ & 2 & & & & 2 \\
\hline \multicolumn{6}{|c|}{ Shared aspects named by two diagnostic groups } \\
\hline Worries/anxiety & & 8 & & 3 & 11 \\
\hline Shame & 3 & & & 2 & 5 \\
\hline $\begin{array}{l}\text { Problems with the } \\
\text { patient role }\end{array}$ & & 1 & & 3 & 4 \\
\hline Loss of control & & & 1 & 1 & 2 \\
\hline \multicolumn{6}{|c|}{ Shared aspects named by three diagnostic groups } \\
\hline $\begin{array}{l}\text { Self-doubts, feelings of } \\
\text { insufficiency }\end{array}$ & 1 & 1 & & 5 & 7 \\
\hline Cognitive impairments & 3 & 1 & 2 & & 6 \\
\hline $\begin{array}{l}\text { Diagnosis perceived } \\
\text { as life-changing } \\
\text { experience }\end{array}$ & & 1 & 2 & 2 & 5 \\
\hline Loss of quality of life & 1 & 1 & 1 & & 3 \\
\hline \multicolumn{6}{|c|}{ Shared aspects named by all four diagnostic groups } \\
\hline Depressive mood & 1 & 2 & 1 & 8 & 12 \\
\hline
\end{tabular}

Numbers represent the total number of mentions and are displayed for each diagnosis individually as well as across all diagnostic groups; grey cells represent categories, which were mentioned at least once in a diagnostic group.

MFS, Marfan syndrome; NF1, neurofibromatosis type 1; PAH, pulmonary arterial hypertension; PSC, primary sclerosing cholangitis.

each diagnostic group as well as the total number across all four transcripts.

\section{Psychological burden}

Participants described different psychological and emotional burdens. In contrast to the medical problems, only few aspects were related to specific diagnoses and we found several shared problems among different diseases. Table 2 shows all topics brought up regarding psychological burden and the number of mentions.

Depressed mood was brought up most frequently and by patients with all four diagnoses. In some cases, participants were diagnosed with a clinically relevant depression, in other cases patients described past or recurring phases of feeling sad and depressed. (Quotations were translated from German and in parts linguistically smoothed to improve readability. After each quotation, the diagnosis and the participant ID is stated. P1 stands for participant 1. IDs were given based on the seating position during the focus groups and have no further meaning. An additional table with quotations for every identified topic can be found in the online supplementary material 1.)

I really had psychological stress or depressive phases, I think that's simply part of it, you have to process, it is like mourning. (Marfan syndrome, P3)

Participants described several symptoms that often are related to depression, for instance rumination and avolition, feelings of insufficiency and self-doubt. In most cases, these feelings were linked to reduced performance and feeling unable to keep up because of the disease. In this context, some patients described feelings of shame. Feelings of shame in another context were reported by patients with neurofibromatosis type 1 because of the visibility of the symptoms. Another aspect frequently experienced across diagnostic groups was anxiety and concern. Participants described mainly future-oriented anxiety concerning the course of the disease or how to deal with the disease at work.

At the beginning there was panic: 'Oh God, what are you doing now? You are only 27, what should you do for a living now? Should you tell your employer or not? Do you keep it a secret? (Marfan syndrome, P1)

Moreover, patients described anxiety and concern related to the outcome of medical examinations.

These constant examinations and this uncertainty, what does it look like now? Every time you go to the ultrasound, is there something malignant? A carcinoma or something? (primary sclerosing cholangitis, P3)

Another shared experience among patients with different diseases was that receiving the diagnosis was perceived as a life-changing experience.

So I actually had a very normal life, it was like that overnight, within a second, what I used to call my life turned around and wasn't what it was before anymore. (Marfan syndrome, P1)

Other common experiences across different diagnoses were a perceived loss of control and loss of quality of life as well as cognitive impairments such as problems focusing or forgetfulness. Moreover, participants reported problems with the patient role. This referred to the tendency of either neglecting to be sick at all or completely defining oneself by the disease. Furthermore, patients described using the disease as an excuse not to deal with other problems.

\section{Problems related to the healthcare system}

Patients with all four diseases described several burdens in relation to the healthcare system. In this category, we found no aspects related to specific diagnoses. All brought up topics were shared by at least three or by all four diagnostic groups. Table 3 provides an overview of these aspects and the number of mentions. 
Table 3 Problems related to the healthcare system and number of mentions (total and by diagnostic group)

\begin{tabular}{llllll}
\hline $\begin{array}{l}\text { Problems related to the } \\
\text { healthcare system }\end{array}$ & NF1 & PSC & PAH & MFS & Total $N$ \\
\hline
\end{tabular}

Shared aspects named by three diagnostic groups

\begin{tabular}{|c|c|c|c|c|c|}
\hline $\begin{array}{l}\text { Problems with } \\
\text { payers/denial of cost } \\
\text { assumption/resistance of } \\
\text { the pension office }\end{array}$ & 6 & 1 & 5 & & 12 \\
\hline $\begin{array}{l}\text { Burden because of } \\
\text { doctor appointments/ } \\
\text { treatments }\end{array}$ & & 3 & & 5 & 8 \\
\hline $\begin{array}{l}\text { Financial burden because } \\
\text { of additional expenditure }\end{array}$ & 1 & 3 & 2 & & 6 \\
\hline \multicolumn{6}{|c|}{ hared aspects named by all four diagnostic groups } \\
\hline $\begin{array}{l}\text { Dissatisfaction with the } \\
\text { professional competence } \\
\text { of caregivers }\end{array}$ & 11 & 6 & 6 & 4 & 27 \\
\hline $\begin{array}{l}\text { Dissatisfaction with } \\
\text { the patient-physician } \\
\text { interaction }\end{array}$ & 7 & 4 & 2 & 8 & 21 \\
\hline $\begin{array}{l}\text { Limited access to } \\
\text { adequate treatment }\end{array}$ & 8 & 7 & 3 & 2 & 20 \\
\hline $\begin{array}{l}\text { Patient feels insufficiently } \\
\text { informed }\end{array}$ & 1 & 5 & 3 & 3 & 12 \\
\hline
\end{tabular}

Numbers represent the total number of mentions and are displayed for each diagnosis individually as well as across all diagnostic groups; grey cells represent categories, which were mentioned at least once in a diagnostic group.

MFS, Marfan syndrome; NF1, neurofibromatosis type 1; PAH, pulmonary arterial hypertension; PSC, primary sclerosing cholangitis.

Patients with primary sclerosing cholangitis and Marfan syndrome described the regularly necessary medical appointments and treatments as burdensome. Individuals with neurofibromatosis type 1 , primary sclerosing cholangitis and pulmonary arterial hypertension reported financial burden because of expenditures not covered by their insurance. In addition, patients stated to suffer from problems with payers such as denial of cost assumption or resistance of the pension office for example, for attaining early retirement. Lack of information about the diseases as well as limited access to adequate care were problems frequently mentioned in all four focus group discussions. The lack of information was described as a result of no information being available, not knowing where to find and how to identify reliable information or physicians not providing sufficient information.

I didn't receive much concrete information about the disease directly from a doctor and because of this information vacuum I approached Doctor Google, even though I know that I have to be very careful. (primary sclerosing cholangitis, $\mathrm{P} 4$ )

I had to wait many months for a proper doctor's appointment, who started to handle it at all. Everyone listened to me but this is now the first one who has finally prescribed something that actually seems to help. (primary sclerosing cholangitis, $\mathrm{P} 4$ )

Other adverse experiences that patients with all four rare diseases had in common were being dissatisfied with either the professional competence of caregivers or with the patient-physician interaction.

I found the contact with physicians partly really critical, I always felt ill when I came from the physician and at other times actually quite well. They were partly very insensitive and for a long time I didn't nearly have the feeling to be medically well cared for. (Marfan syndrome, P4)

Dissatisfaction with the professional competence of caregivers was often described as a problem resulting from a lack of knowledge about the conditions.

Even experts, that is, doctors, are not familiar with this disease in detail and then still have to give advice and one thinks, that's an authority person, that's a doctor, he has to know it but they don't even know what primary sclerosing cholangitis is. (primary sclerosing cholangitis, $\mathrm{P} 3$ )

\section{Constraints}

Constraints in different areas of patients' lives were brought up in all four focus groups with all topics being shared by at least two diagnostic groups. We found no aspects related to specific diagnoses. The results are displayed in table 4.

The constraints partly referred to functional restrictions, for instance not being able to take care of the household.

I can't do anything in the household because I can't walk. I have to let someone do that. I can't even hang out my laundry in winter. The drying loft is on the fifth floor, I can't even get there. (pulmonary arterial hypertension, P1)

Table 4 Constraints and number of mentions (total and by diagnostic group)

\begin{tabular}{llllll} 
Constraints & NF1 & PSC & PAH & MFS & Total $N$ \\
\hline
\end{tabular}

Shared aspects named by two diagnostic groups

$$
\text { Constraints in daily life } \quad 3 \quad 8
$$

Shared aspects named by all four diagnostic groups

\begin{tabular}{l|lllll|}
$\begin{array}{l}\text { Constraints in personal } \\
\text { and social life }\end{array}$ & 4 & 9 & 7 & 5 & 25 \\
$\begin{array}{l}\text { Constraints in } \\
\text { professional life }\end{array}$ & 9 & 4 & 3 & 6 & 22 \\
\hline
\end{tabular}

Numbers represent the total number of mentions and are displayed for each diagnosis individually as well as across all diagnostic groups; grey cells represent categories, which were mentioned at least once in a diagnostic group.

MFS, Marfan syndrome; NF1, neurofibromatosis type 1; PAH, pulmonary arterial hypertension; PSC, primary sclerosing cholangitis. 
Table 5 Interpersonal problems and number of mentions (total and by diagnostic group)

\begin{tabular}{|c|c|c|c|c|c|}
\hline Interpersonal problems & NF1 & PSC & РAH & MFS & Total $N$ \\
\hline \multicolumn{6}{|c|}{ Shared aspects named by two diagnostic groups } \\
\hline Burden for close others & 1 & & & 6 & 7 \\
\hline $\begin{array}{l}\text { Problems setting } \\
\text { boundaries }\end{array}$ & 2 & & & 4 & 6 \\
\hline \multicolumn{6}{|c|}{ Shared aspects named by three diagnostic groups } \\
\hline $\begin{array}{l}\text { Stigmatisation/ } \\
\text { discrimination }\end{array}$ & 13 & & 1 & 2 & 16 \\
\hline $\begin{array}{l}\text { Lack of social/emotional/ } \\
\text { psychological support }\end{array}$ & & 1 & 3 & 4 & 8 \\
\hline $\begin{array}{l}\text { The way others deal with } \\
\text { the disease is perceived } \\
\text { as counterproductive }\end{array}$ & 1 & & 4 & 3 & 8 \\
\hline $\begin{array}{l}\text { Illness is invisible for } \\
\text { others }\end{array}$ & 1 & & 1 & 1 & 3 \\
\hline \multicolumn{6}{|c|}{ Shared aspects named by all four diagnostic groups } \\
\hline $\begin{array}{l}\text { Others lack } \\
\text { understanding of the } \\
\text { disease }\end{array}$ & 6 & 5 & 5 & 6 & 22 \\
\hline
\end{tabular}

Numbers represent the total number of mentions and are displayed for each diagnosis individually as well as across all diagnostic groups; grey cells represent categories, which were mentioned at least once in a diagnostic group.

MFS, Marfan syndrome; NF1, neurofibromatosis type 1; PAH, pulmonary arterial hypertension; PSC, primary sclerosing cholangitis.

Constraints in the professional life were brought up by patients with all four diagnoses. Some patients described frequent doctoral appointments to conflict with working hours. Other participants reported strains by trying to hide the disease at work in order not to be discriminated. The majority of the work-related problems referred to the disease negatively affecting the ability to work. In some cases, this resulted in permanent occupational disability.

Moreover, patients with all four diagnoses mentioned constraints affecting their personal life. This includes restrictions regarding vacations or hobbies. Patients described the diseases to affect long-term decisions and the realisation of plans and wishes, for instance concerning family planning.

What burdens me the most is can I pass this on or not. What about family planning? Am I even capable of raising a child? If it comes into the world healthy at all. (primary sclerosing cholangitis, $\mathrm{P} 4$ )

\section{Interpersonal problems}

Interpersonal problems were experienced in all diagnostic groups. In this category, we found no aspects related to specific diagnoses. Patients reported a variety of shared aspects, which are displayed in table 5 .

All four diagnostic groups shared the experience of others lacking understanding of the disease. Furthermore, participants reported disbelief by others and having to exculpate themselves about being sick.

Every time you meet this person who says 'why don't you listen to me? I told you to just take this pill or to change the doctor or something' it was hurtful for me because they just didn't believe me that I do everything, which is right in my eyes. (pulmonary arterial hypertension, $\mathrm{P} 4$ )

Another problem related to the social environment was a lack of support.

There are friends who just can't handle the fact that you're sick. They find it very annoying that I deal relatively open with having an illness and that it usually doesn't have a great prognosis. That quickly separates the wheat from the chaff. There is a small group left, with whom you can talk about it, who can be a support. (primary sclerosing cholangitis, P2)

Moreover, patients reported to suffer from stigmatisation. Stigmatisation was mainly related to either having a disease in general or to the visibility of the symptoms. In many cases, patients experienced discrimination and mobbing by colleagues, who labelled patients lazy or unproductive. Being stigmatised because of the visibility of the symptoms was frequently mentioned by individuals with neurofibromatosis type 1 . The experienced stigmatisation ranges from people staring in public transit to being banned from public facilities such as swimming pools.

I was banned from swimming pools because other customers complained because of my outer appearance. That's tough to cope with. (neurofibromatosis type $1, \mathrm{P} 3)$

One patient who works with children described experiences of parents not wanting her to care for their children.

With my outer appearance, the children are often taken away from me by their parents. I shall not care for their children and they won't even talk to me, they go to my colleagues and make them bring their child to bed. (neurofibromatosis type 1, P3)

At the same time, the invisibility of the disease can be perceived as burdensome, too. This was brought up by patients with neurofibromatosis type 1, pulmonary arterial hypertension and Marfan syndrome.

If you have something like a broken leg, everyone understands you, but when I get on the bus and say: 'I absolutely have to sit down', everyone says: 'But you're still young'. Nobody understands it, because you just can't see it. (Marfan syndrome, P2)

Another interpersonal issue reported by individuals with neurofibromatosis type 1 and Marfan syndrome was problems setting boundaries, for example, agreeing to social events despite physical exhaustion. Moreover, some patients perceived the way others deal with the disease as 
counterproductive. In some cases, this related to people giving unwanted advice. In other cases, this referred to the behaviour of patients' parents. This was specifically the case in neurofibromatosis type 1 and Marfan syndrome because both conditions are genetically caused and therefore likely to emerge in childhood. In this context, individuals with neurofibromatosis type 1 and Marfan syndrome additionally reported that their disease was often burdensome for their parents.

My parents were so afraid out of ignorance, that they overprotected me, that I couldn't do anything by myself, couldn't gather any experiences. I wish more parents would learn how to support their child in order to be able to live a normal life. (neurofibromatosis type $1, \mathrm{P} 3$ )

\section{Summary of commonalities and differences between the four diagnostic groups}

Taken together, the majority of medical problems seemed to be specific for certain diseases with few exceptions such as pain or diarrhoea. Contrarily, many shared burdens were found regarding psychological and interpersonal problems, healthcare aspects and constraints. Topics brought up in all four diagnostic groups were depressed mood, limited access to adequate treatment, feeling insufficiently informed, dissatisfaction with the professional competence of caregivers and patient-physician interaction, constraints in professional and in personal/social life and others lacking understanding for the disease. To further illustrate, which aspects are shared among different diagnostic groups, we attached word clouds for every main category with colour and size of each topic indicating the number of diagnostic groups who named it in the online supplementary material 2.

\section{DISCUSSION}

This study aimed to examine perceived burdens of living with rare chronic diseases and identify commonalties and differences in the experiences of patients with four heterogeneous conditions. Patients with neurofibromatosis type 1, primary sclerosing cholangitis, pulmonary arterial hypertension and Marfan syndrome described medical problems, psychological burdens, constraints in professional or personal life, problems related to the healthcare system and interpersonal problems. To our knowledge, there are no qualitative studies directly comparing the experiences of patients with heterogeneous chronic conditions in order to identify differing and shared aspects.

The majority of the medical problems were specific for certain conditions and therefore reflect the clinical heterogeneity of the four diseases. Some of the psychosocial problems seem to be related to specific medical symptoms of certain diseases. Specific for diseases usually emerging in childhood (neurofibromatosis type 1, Marfan syndrome) was parental behaviour perceived as overprotective and burden for patients' parents. This is in line with other qualitative studies on parental experiences with paediatric rare diseases such as osteogenesis imperfecta, ${ }^{11}$ inherited metabolic diseases ${ }^{12}{ }^{13}$ and Zellweger spectrum disorders ${ }^{14}$ from a caregivers' perspective. In these studies, parents of affected children reported to experience concern, emotional stress and feelings of uncertainty and being overwhelmed, which stresses the need for support systems for caregivers of patients with paediatric rare diseases. Another disease-specific experience was stigmatisation and discrimination because of the visibility of the symptoms. In contrast, the invisibility of a disease can cause strain, too. This finding is in line with other studies on the impact of illness visibility showing that both visibility ${ }^{15}$ and invisibility ${ }^{1617}$ of a disease can be perceived as burdensome.

Contrarily to the heterogeneous disease-specific problems, we found many shared experiences across diagnostic groups including psychological burdens (e.g., depressive mood), constraints (e.g., in professional and personal life) and interpersonal problems (e.g., stigmatisation and others lacking understanding of the disease). Patients with four heterogeneous conditions independently brought up a variety of common topics. This indicates that despite the clinical heterogeneity of rare diseases, affected individuals face many similar problems. This result is consistent with a systematic review of qualitative studies with each focusing on a specific single rare disease or group of rare diseases demonstrating that patients described similar problems. ${ }^{5}$ In a quantitative study by our working group assessing depression and anxiety in patients with different rare diseases, we came to a similar conclusion showing that psychopathology is mainly not associated with diagnosis-related aspects, but rather with cross-diagnostic aspects such as illness perceptions. ${ }^{2}$

A subset of the shared burdens that patients described have been found in studies on more common chronic conditions as well. In qualitative studies on problems related to diabetes type 1 for instance, patients described the disease conflicting with the professional $\operatorname{life}^{18}$ as well as experiences of stigmatisation and others lacking understanding. ${ }^{19} 20$ In quantitative studies on patients with common chronic conditions, patients reported decreased quality of life, loss of control, constraints and depression or anxiety symptoms. ${ }^{21-24}$ Being affected by a rare chronic condition thus might in part resemble the experiences other patients with more common chronic conditions have. In addition to the universal experience of patients affected by chronic conditions, some of the described problems seem to be linked to the low prevalence and therefore might be specific for rare diseases. The majority of these problems pertain to the healthcare system, for example, limited access to adequate treatment and physicians lacking expertise and knowledge, which results in dissatisfaction with the professional competence of caregivers. Patients further described to feel insufficiently informed about their disease. Other aspects, which may be a consequence of limited knowledge about 
the diseases as well, are related to the social environment, for example, others lacking understanding for the patients. These experiences might express the unique challenges of patients with rare diseases and furthermore were aspects shared by patients with all four diseases.

The shared aspects among heterogeneous conditions can help develop generic interventions in order to provide adequate psychosocial support. This is particularly useful for patients with rare diseases since offering disease-specific support for every single rare disease is not realistic. Many of the common problems found in the present qualitative study are related to the healthcare system. Similar problems reflecting the difficulties of providing adequate care for patients with rare diseases have been showed from a healthcare perspective. In a recent quantitative study, Jo and collegues found that primary care providers are more likely to refer patients with rare diseases to other providers, which may reflect a lack of knowledge and insecurity on how to adequately care for the patients. ${ }^{25}$ Central contact points such as specialised care centres for rare diseases can facilitate access to adequate treatment. Creating supraregional and interdisciplinary networks could help exchange existing knowledge and expertise. A well-organised cooperation and coordination between experts, patients and relatives could increase satisfaction with medical care. Insufficient knowledge about the conditions as a consequence of the low prevalence could be an underlying key aspect of the unique challenges patients with rare diseases are confronted with. Clinicians could try to target patients' high information need in consultations and help patients find reliable medical information about their disease.

This study has some limitations. We recruited patients partly via specialised care centres for the specific diseases at the University Medical Center Hamburg-Eppendorf. Consequently, our participants likely received specialised care by experts for their diseases. Experiences of patients not having access to such specialised treatment might be different. Moreover, we included patients with four specific rare chronic diseases, which does not allow generalisation to other rare diseases. Furthermore, we conducted one focus group per diagnosis. An advantage of this procedure was that patients did not have to explain their condition to other participants. An alternative procedure with focus groups with patients with different diseases may have promoted further discussion about commonalities across diagnoses. We, in contrast, identified commonalities between groups based on content analysis. In our study, sample sizes were relatively small (four to five patients per diagnostic group) and we did not collect data until reaching data saturation. It is unclear whether further focus groups with other participants would have brought up additional topics. However, considerations about information power imply that a small sample size might be adequate in our case: Malterud et al argue that a narrow study aim within a specific patient population is associated with a higher information power and therefore allows smaller sample sizes. ${ }^{26}$ Furthermore, the focus groups were held by researchers experienced in leading group discussions and following an interview guide, which was thoroughly developed beforehand. Both aspects foster a high quality of dialogue between researchers and participants, which promotes information power, too. ${ }^{26}$ Given that the study population is by definition rare, working with small sample sizes for studies that require the presence of participants allows to study this population at all. Future research could study larger samples by implementing technological measures that allow participation from home. Finally, even though our results indicate that there are unique challenges when dealing with a rare chronic disease, we did not directly contrast our sample with patients with more common chronic conditions. A direct comparison of rare and more common chronic conditions could help clarify the impact of the low prevalence.

\section{CONCLUSION}

Our results show that living with a rare chronic disease causes substantial burden beyond medical symptoms. Some of the experiences seem to be specifically linked to the rarity of the conditions. This illustrates that there are unique challenges when dealing with a chronic disease with a low prevalence. Lack of knowledge, even among healthcare professionals, could be one of the key aspects underlying these unique challenges.

To our knowledge, this is the first qualitative study explicitly trying to identify common and differing experiences of patients with heterogeneous rare diseases. Our results show that while medical problems differ hugely between diseases, patients reported many similar experiences concerning psychological and interpersonal problems, healthcare aspects and constraints. Despite the heterogeneity of different rare diseases, patients face many common problems. Addressing these commonalities in interventions could help provide support for patients with different rare diseases concurrently. In conclusion, support approaches targeted to the specific needs of patients with rare diseases, which at the same time are applicable for different rare diseases concurrently, might be the most adequate way to provide comprehensive healthcare for this patient population in the face of finite healthcare resources.

Acknowledgements This study is part of the project 'Patients for patients: qualified peer counselling and self-management for patients with rare chronic diseases', a research project aiming to develop and evaluate a self-management programme for patients with different rare chronic diseases. We would like to thank our partners ACHSE e.V. and the Martin Zeitz Center for Rare Diseases at the University Medical Center Hamburg-Eppendorf for their cooperation and support. We are grateful to all patients who supported us by participating in the study. We would further like to thank Anne Marie-Waßmuth (AW), Laura Holz (LH) and Johanna Dreyer (JD) for supporting data analysis. Moreover, we would like to thank the editors and reviewers for the time they invested in reading our manuscript and for the constructive feedback.

Contributors All authors have contributed to conception, design, acquisition, analysis or interpretation of this study. Further, all authors contributed to writing or editing the draft of the manuscript and approved to the final version. Finally, all 
authors agree to be accountable for all aspects of the work. NU: conceptualisation, methodology, formal analysis, investigation, data curation, writing: original draft, writing: review and editing, visualisation. BL: conceptualisation, resources, writing: review and editing, supervision, project administration, funding acquisition. MKD: conceptualisation, methodology, formal analysis, investigation, data curation, writing: review and editing, supervision, project administration, funding acquisition.

Funding This research project is funded by the Robert Bosch Stiftung.

Disclaimer The foundation provided funding in the form of salaries for authors (NU) and research materials but did not play a role in the study design, data collection and analysis, decision to publish or preparation of the manuscript.

Competing interests None declared.

Patient consent for publication Not required.

Ethics approval Ethics approval was given by the independent ethics committee of the Hamburg Medical Chamber (Ärztekammer Hamburg) on 2 February 2016 (reference number PV5088).

Provenance and peer review Not commissioned; externally peer reviewed.

Data availability statement Data are available upon reasonable request. Data cannot be made publicly available due to ethical reasons. In the area of rare diseases, information about the diagnosis in combination with personal information may compromise anonymity and confidentiality of the participants. The independent ethics committee of the Hamburg Medical Chamber assessed our research project beforehand. The ethics vote allows sharing data with eligible researchers but we do not have approval to share the data publicly. Researchers interested in getting access to the data should feel free to contact the corresponding author ( $\mathrm{NU}-\mathrm{n}$. uhlenbusch@uke.de) or the principal investigator (BL-b.loewe@uke.de).The ethics committee can be contacted at: ethik@aekhh.de.

Open access This is an open access article distributed in accordance with the Creative Commons Attribution Non Commercial (CC BY-NC 4.0) license, which permits others to distribute, remix, adapt, build upon this work non-commercially, and license their derivative works on different terms, provided the original work is properly cited, appropriate credit is given, any changes made indicated, and the use is non-commercial. See: http://creativecommons.org/licenses/by-nc/4.0/.

\section{ORCID iD}

Natalie Uhlenbusch http://orcid.org/0000-0002-4031-9839

\section{REFERENCES}

1 Eurordis. What is a rare disease? : EURORDIS - Rare Diseases Europe, 2017. Available: http://www.eurordis.org/sites/default/files/ publications/Fact_Sheet_RD.pdf [Accessed 4 Mar 2019].

2 Uhlenbusch N, Löwe B, Härter M, et al. Depression and anxiety in patients with different rare chronic diseases: a cross-sectional study. PLoS One 2019;14:e0211343.

3 Katon W, Lin EHB, Kroenke K. The association of depression and anxiety with medical symptom burden in patients with chronic medical illness. Gen Hosp Psychiatry 2007;29:147-55.

4 Lichtman JH, Froelicher ES, Blumenthal JA, et al. Depression as a risk factor for poor prognosis among patients with acute coronary syndrome: systematic review and recommendations: a scientific statement from the American heart association. Circulation 2014:129:1350-69.

5 von der Lippe C, Diesen PS, Feragen KB. Living with a rare disorder: a systematic review of the qualitative literature. Mol Genet Genomic Med 2017:5:758-73.

6 Orphanet. Neurofibromatosis type 1: Orpha.net, 2014. Available: https://www.orpha.net/consor/cgi-bin/Disease_Search.php? Ing=EN\&data_id=185\&Disease_Disease_Search_diseaseGroup= neurofibromatosis-type-1\&Disease_Disease_Search_diseaseType= Pat\&Disease(s)/groupofdiseases=Neurofibromatosis-type-1\&title= Neurofibromatosistype1\&search=Disease_Search_Simple [Accessed 4 Mar 2019].

7 Orphanet. Primary sclerosing cholangitis: Orpha.net, 2017. Available: https://www.orpha.net/consor/cgi-bin/Disease_Search.php?lng=
EN\&data_id=783\&Disease_Disease_Search_diseaseGroup=primarysclerosing-cholangitis\&Disease_Disease_Search_diseaseType= Pat\&Disease(s)/groupofdiseases=Primary-sclerosing-cholangitis\& title=Primarysclerosingcholangitis\&search=Disease_Search_Simple [Accessed 4 Mar 2019].

8 Orphanet. Pulmonary arterial hypertension: Orpha.net, 2015. Available: https://www.orpha.net/consor/cgi-bin/Disease Search.php? Ing=EN\&data_id=18210\&Disease_Disease_Search_ diseaseGroup=Pulmonary-arterial-hypertension\&Disease_Disease_ Search_diseaseType=Pat\&Disease(s)/groupofdiseases=Pulmonaryarterial-hypertension\&title=Pulmonaryarterialhypertension\&search $=$ Disease Search Simple [Accessed 4 Mar 2019].

9 Orphanet. Marfan syndrome: Orpha.net, 2010. Available: https:// www.orpha.net/consor/cgi-bin/Disease_Search.php?lng=EN\& data $i d=109 \&$ Disease Disease Search diseaseGroup=marfansyndrome\&Disease_Disease_Search_diseaseType=Pat\&Disease(s)/ groupofdiseases=Marfan-syndrome\&title=Marfansyndrome\&search $=$ Disease_Search_Simple [Accessed 4 Mar 2019].

10 Mayring P, Fenzl T. Qualitative inhaltsanalyse [Qualitative content analysis]. In: Baur N, Blasius J, eds. Handbuch methoden der empirischen sozialforschung [Handbook methods of empirical social research]. Wiesbaden: Springer, 2014: 543-56.

11 Dogba MJ, Rauch F, Tre G, et al. Shaping and managing the course of a child's disease: parental experiences with osteogenesis imperfecta. Disabil Health J 2014;7:343-9.

12 Khangura SD, Tingley K, Chakraborty P, et al. Child and family experiences with inborn errors of metabolism: a qualitative interview study with representatives of patient groups. J Inherit Metab Dis 2016;39:139-47.

13 Siddiq S, Wilson BJ, Graham ID, et al. Experiences of caregivers of children with inherited metabolic diseases: a qualitative study. Orphanet J Rare Dis 2016;11:168.

14 Bose M, Mahadevan M, Schules DR, et al. Emotional experience in parents of children with Zellweger spectrum disorders: a qualitative study. Mol Genet Metab Rep 2019;19.

15 Olney MF, Brockelman KF. The impact of visibility of disability and gender on the self-concept of university students with disabilities. $J$ Postsecond Educ Disabil 2005;18:80-91.

16 Sumpton D, Thakkar V, O'Neill S, et al. "It's not me, it's not really me." Insights from patients on living with systemic sclerosis: An interview study. Arthritis Care Res (Hoboken) 2017:69:1733-42.

17 Ciribassi RM, Patil CL. "We don't wear it on our sleeve": Sickle cell disease and the (in)visible body in parts. Soc Sci Med 2016;148:131-8.

18 Hansen UM, Cleal B, Willaing I, et al. Managing type 1 diabetes in the context of work life: a matter of containment. Soc Sci Med 2018;219:70-7.

19 Browne JL, Ventura A, Mosely K, et al. 'I call it the blame and shame disease': a qualitative study about perceptions of social stigma surrounding type 2 diabetes. BMJ Open 2013;3:e003384.

20 Browne JL, Ventura A, Mosely K, et al. 'I'm not a druggie, I'm just a diabetic': a qualitative study of stigma from the perspective of adults with type 1 diabetes. BMJ Open 2014;4:e005625.

21 Alonso J, Ferrer M, Gandek B, et al. Health-related quality of life associated with chronic conditions in eight countries: results from the International quality of life assessment (IQOLA) project. Qual Life Res 2004;13:283-98.

22 Kessler RC, Ormel J, Demler O, et al. Comorbid mental disorders account for the role impairment of commonly occurring chronic physical disorders: results from the National comorbidity survey. $J$ Occup Environ Med 2003;45:1257-66.

23 Sareen J, Jacobi F, Cox BJ, et al. Disability and poor quality of life associated with comorbid anxiety disorders and physical conditions. Arch Intern Med 2006;166:2109-16.

24 Clarke DM, Currie KC. Depression, anxiety and their relationship with chronic diseases: a review of the epidemiology, risk and treatment evidence. Med J Aust 2009;190:54-60.

25 Jo A, Larson S, Carek P, et al. Prevalence and practice for rare diseases in primary care: a national cross-sectional study in the USA. BMJ Open 2019;9:e027248.

26 Malterud K, Siersma VD, Guassora AD. Sample size in qualitative interview studies: guided by information power. Qual Health Res 2016;26:1753-60. 